\title{
- Fluidoterapia em grandes animais - Parte II: quantidade e vias de administração
}

\section{- Fluid therapy in large animals - Part II: amount and administration routes}

\section{* Antônio Cézar de Oliveira Dearo ${ }^{1}$ - CRMV-PR - nº 4428 Peter Reichmann ${ }^{2}$ - CRMV-PR - n 2028}

1 Professor Assistente do Departamento de Clínicas Veterinárias da UEL/PR.

2 Professor Adjunto do Departamento de Clínicas Veterinárias da UEL/PR.

*Universidade Estadual
de Londrina CCA - DCV
Caixa Postal 6001
CEP: $86051-990$
Londrina - Paraná
Fone: $(0 * \star 43)$ 371-4269/371-4329
End. Eletrôn.: dearoaco@uel.br

\section{RESUMO}

Tão importante quanto a indicação de reposição hidroeletrolítica e a escolha da solução mais apropriada é o conhecimento da quantidade de fluidos, das vias de administração e da velocidade de reposição a serem utilizadas. Considerando que em muitas situações a fluidoterapia deve estender-se por períodos prolongados, o uso de cateteres intravenosos, associados a sistemas que permitem fluxo contínuo durante várias horas do dia, reduz em muito a necessidade de acompanhamento ininterrupto.

Palavras-chave: fluidoterapia, desidratação, cateter, hidratação, eletrólitos.

\section{Quantidade de Fluidos e Vias de Administração}

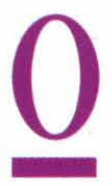

plano de reposição hidroeletrolítica deve incluir não só a escolha da solução mais adequada como também sua quantidade, via de administração e velocidade de reposição. Como já referido na parte I deste artigo, todos esses fatores estão na dependência do quadro clínico e do grau de desidratação apresentado.

O volume total de líqüido a ser administrado deve incluir fundamentalmente a quantidade de fluidos perdida pela desidratação e as necessidades diárias de manutenção (GROSS, 1992; SCHMALL, 1997). O déficit de fluido perdido pode ser calculado multiplicando-se a porcentagem estimada de desidratação pelo peso do animal em quilo. Já as necessidades de manutenção são variáveis de acordo com a idade, atividade e temperatura ambiente. Animais jovens possuem maiores necessidades de manutenção em relação a animais adultos. De forma geral, emprega-se a quantidade de 50 a 100 $\mathrm{ml} / \mathrm{kg} / \mathrm{dia}$, utilizando-se os valores inferiores para animais adultos e os superiores para animais jovens (SEAHORN; CORNICK-SEAHORN, 1994; SCHMALL, 1997). Imaginando-se como exemplo um cavalo de 400 $\mathrm{kg}$, portador de cólica e que apresenta um grau de desidratação clínica estimada em $8 \%$, o seguinte cálculo deve ser realizado para se determinar a quantidade total de fluidos a ser administrada num período de 24 horas: 
DEARO, A. C. O.; REICHMANN, P. Fluidoterapia em grandes animais - Parte II: quantidade e vias de administração / Fluid therapy in large animals - Part II: amount and administration routes / Rev. educ. contin. CRMV-SP / Continuous Education Journal CRMV-SP, São Paulo, volume 4, fascículo 3, p. 3 - 11, 2001.

Volume total $=\%$ desidratação $\times$ peso $(\mathrm{kg})+$ manutenção $(50 \mathrm{ml} / \mathrm{kg})$

$$
\begin{aligned}
& \text { Volume total }=(8 \% \times 400)+(50 \times 400) \\
& \text { Volume total }=32(\mathrm{I})+20000(\mathrm{ml}) \\
& \text { Volume total }=32(\mathrm{I})+20(\mathrm{I})=52 \text { litros. }
\end{aligned}
$$

Tomando-se agora como exemplo um bezerro neonato de $50 \mathrm{~kg}$ portador de diarréia e desidratação clínica estimada em $10 \%$, tem-se:

$$
\begin{aligned}
& \text { Volume total }=(10 \% \times 50)+(100 \times 50) \\
& \text { Volume total }=5+5=10 \text { litros. }
\end{aligned}
$$

Em grandes animais, as vias mais comuns utilizadas para a administração de fluidos é a oral e a intravenosa. Outras vias, como a intraperitoneal e a retal, podem ser utilizadas, porém são empregadas apenas em casos específicos (GROSS, 1992). Em potros neonatos a via intra-óssea pode ser usada como via alternativa à via intravenosa (GOLENZ et al., 1993). A via subcutânea não é utilizada em grandes animais em razão do grande volume que comumente é administrado nessas espécies (GROSS, 1992). A via oral é, sem dúvida nenhuma, a mais segura. Além de permitir a administração de gran- des volumes de líquidos e eletrólitos em um curto espaço de tempo é de baixo custo. Entretanto, para que possa ser utilizada é necessário que as funções de transporte e absorção intestinais não estejam comprometidas. Uma situação prática em que essas funções apresentam-se alteradas pode ser exemplificada por cavalos com cólica que apresentam grandes quantidades de refluxo pela sonda nasogástrica (MORRIS, 1988; SPURLOCK; WARD, 1990; SCHMALL, 1992, 1997; BARTON; MOORE, 1999).

Na prática, a via oral tem sua maior indicação em situações clínicas de desidratação decorrentes de exercícios físicos, anorexia, diarréias e cólica em eqüinos provocada por compactação no cólon maior (MORRIS, 1988; ROUSSEL Jr.; KASARI, 1990; SCHMALL, 1992; McGINNESS et al., 1996; LOPES et al., 1998).

Em eqüinos adultos, a administração é realizada a cada 30-60 minutos por meio de sonda nasogástrica e a quantidade não deve ser superior a 5 a 10 litros (SCHMALL, 1992, 1997). Em bovinos e pequenos ruminantes, utiliza-se uma sonda orogástrica, a qual é introduzida por meio de um guia de sonda metálico em forma de cilindro posicionado na cavidade oral.

Preferencialmente devem ser administradas soluções hipotônicas ou isotônicas (ROSE, 1981).

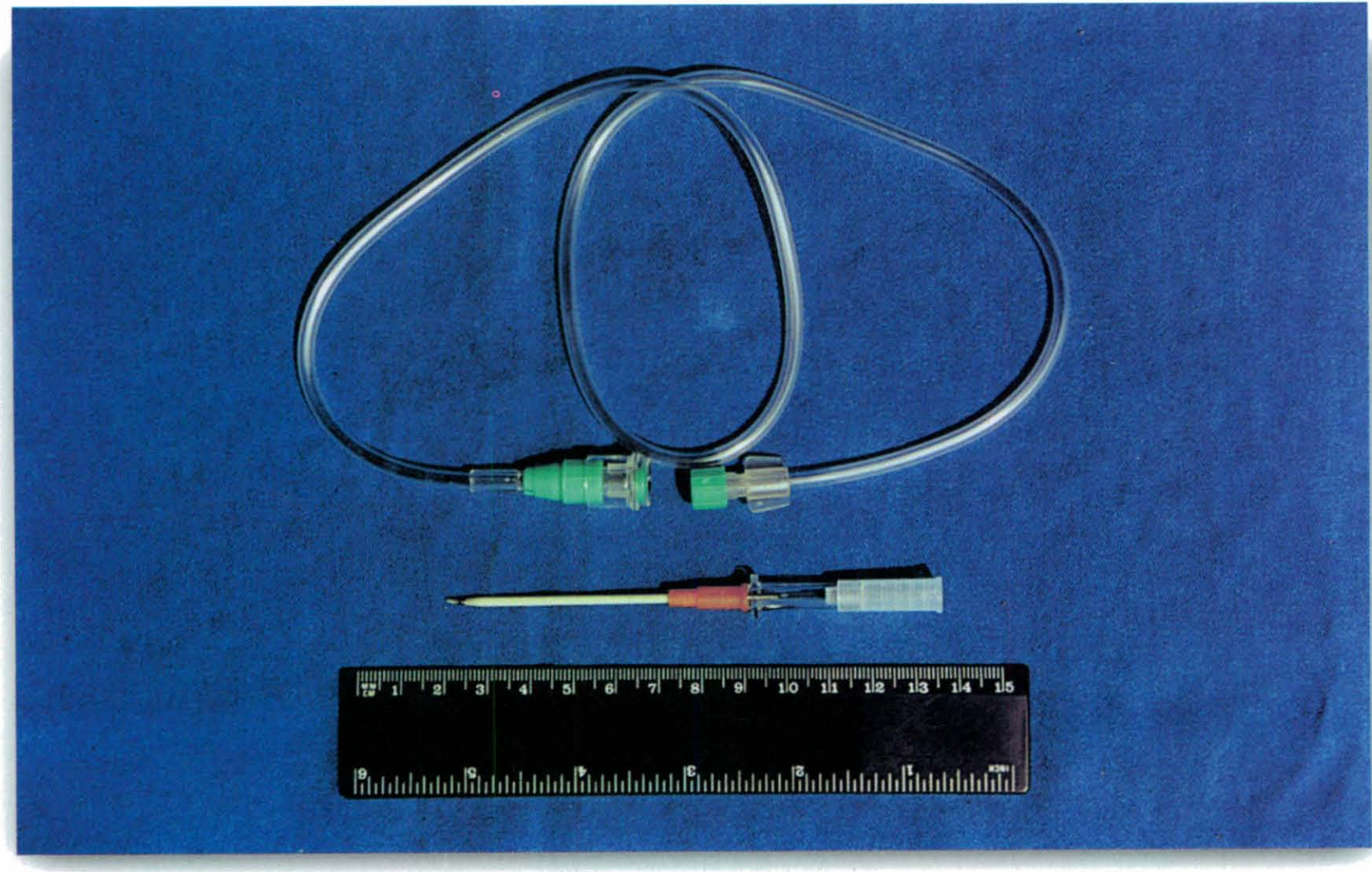

Figura 1. Cateter $14 \mathrm{G}$ x $1.88 \mathrm{IN}$ e tubo extensor. 
DEARO, A. C. O.; REICHMANN, P. Fluidoterapia em grandes animais - Parte II: quantidade e vias de administração / Fluid therapy in large animals - Part II: amount and administration routes / Rev. educ. contin. CRMV-SP / Continuous Education Journal CRMV-SP, São Paulo, volume 4, fascículo 3, p. 3 - 11, 2001

A via intravenosa é a de eleição para a fluidoterapia na grande maioria das situações clínicas, principalmente quando se deseja a administração de grandes volumes e de forma rápida.

Nos últimos anos, inúmeros problemas relativos ao uso prolongado da via intravenosa em animais, notadamente no que diz respeito à "manutenção da veia", puderam ser solucionados pelo uso de cateteres intravenosos. A principal vantagem de sua utilização reside na manutenção prolongada do acesso intravenoso, suprimindo dessa forma, a necessidade de repetidas e inconvenientes venopunções que, em última análise, são as principais responsáveis por danos vasculares extensos e, muitas vezes, irreversíveis (WILLIAMSON, 1988; DORNBUSCH et al., 2000).

A escolha do cateter mais adequado deve levar em consideração o porte do animal, a velocidade de administração, o tempo de permanência, o custo e a capacidade trombogênica do cateter, determinada fundamentalmente pelo seu diâmetro e material de fabricação (SPURLOCK; WARD, 1990; SEAHORN; CORNICKSEAHORN, 1994). Materiais como silicone e poliuretano são os que apresentam menor atividade trombogênica (SPIER et al., 1990; GARDNER; DONAWICK, 1992; SCHMALL, 1997)

Os cateteres mais usados em grandes animais são os de diâmetro $14 \mathrm{G}$ e $16 \mathrm{G}$. Sempre que possível, deve-se utilizar o cateter de menor diâmetro, pois quanto menor o trauma vascular, menores serão as possibilidades de ocorrência de complicações (Figura 1).

O conhecimento da maneira correta de introdução e manutenção do cateter são vitais para a minimização dos danos vasculares e a maximização do tempo de uso do cateter. A introdução deve ser realizada de forma asséptica mediante o uso de luvas estéreis após tricotomia e anti-sepsia cirúrgica do local. O sentido de introdução deve seguir o sentido do fluxo sangüíneo. A prévia injeção subcutânea de 0,5 a $1 \mathrm{ml}$ de lidocaína no local de perfuração facilita a manobra, especialmente em animais inquietos ou de difícil temperamento. Caso seja necessário e a situação clínica em questão permitir, deve-se sedar o animal para se proceder à introdução.

Após a venopunção inicial, realizada em ângulo de $45^{\circ}$ em relação à veia e verificada pela presença de sangue no bulbo do mandril, deve-se proceder à introdução conjunta do cateter e do mandril por cerca de mais 1,5 $\mathrm{cm}$ aproximadamente (Figuras 2 a 4). Em seguida, devese manter o mandril imóvel e deslizar somente o cateter para o interior da veia, mantendo-se o mandril apenas como guia (WILLIAMSON, 1988; TRAUB-DARGATZ, 1999) (Figura 5). Após a remoção do mandril, acoplar ao cateter o tubo extensor* já conectado ao equipo (Figuras 6 e 7). A ausência de fluxo sangüíneo após a retirada do mandril é indicativa de mal posicionamento do cateter, muitas vezes decorrente de dobras que ocorrem durante a introdução pelo tecido subcutâneo. Nessas circunstâncias deve-se retirar o cateter. A tentativa de reintrodução do mandril "às cegas" usualmente resulta em perfuração do cateter no local da dobradura e trauma adicional aos tecidos circunvizinhos.

A fixação do cateter à pele pode ser feita com cola à base de cianoacrilato (ex: superbonder®), porém é melhor realizada por meio de suturas de fixação do tubo extensor à pele em dois únicos pontos intervalados por 1 a $2 \mathrm{~cm}$, dos quais o primeiro aplicado imediatamente atrás da conexão em rosca do tubo extensor ao cateter (Figura 8). Completada a fixação do conjunto, aplicase pequena quantidade de pomada antimicrobiana na junção do cateter com a pele.

Terminada a fluidoterapia, deve-se heparinizar todo o conjunto (cateter e tubo extensor), tomando-se o cuidado de não permitir a entrada de ar no sistema (Figura 9). A extremidade livre do tubo extensor pode ser presa à crina do cavalo quando a veia utilizada for a jugular. Preferencialmente, deve-se recobrir todo o conjunto por meio de bandagem adesiva (Figura 10). Outras veias, como a torácica lateral, a cefálica e a safena, podem ser utilizadas, porém, nelas há uma maior dificuldade quanto à manutenção do cateter (BAYLY; VALE, 1982; SPURLOCK; WARD, 1990; SEAHORN; CORNICK-SEAHORN, 1994; TRAUB-DARGATZ, 1999).

A obstrução do cateter, que muitas vezes decorre da coagulação do sangue no interior do conjunto, pode ser evitada mediante a "lavagem" de todo o sistema com uma solução heparinizada (10 UI de heparina/ml de solução salina), a cada 6 horas (WILLIAMSON, 1988; GARDNER; DONAWICK, 1992; TRAUB-DARGATZ, 1999). A "lavagem" de todo conjunto também deve ser realizada antes e após a aplicação de qualquer medicação pelo cateter. Situações em que duas ou mais medicações são aplicadas no mesmo horário, deve-se realizar a "lavagem" após a aplicação de cada droga separadamente, de forma a impedir a permanência de resíduos no interior do conjunto e, com isso, eventuais reações de incompatibilidade (TRAUB-DARGATZ, 1999).

Caso ocorra a obstrução, remover o cateter, substituir o conjunto e proceder nova introdução numa outra veia. Cateteres obstruídos pela coagulação do sangue no seu interior jamais devem ser desobstruídos mediante a injeção sob pressão de solução heparinizada. Além de

* Extensofix $60 \mathrm{~cm}$ - Laboratórios B. Braun S.A. 
DEARO, A. C. O.; REICHMANN, P. Fluidoterapia em grandes animais - Parte II: quantidade e vias de administração / Fluid therapy in large animals - Part II: amount anc administration routes / Rev. educ. contin. CRMV-SP / Continuous Education Journal CRMV-SP, São Paulo, volume 4, fascículo 3, p. 3 - 11, 2001.

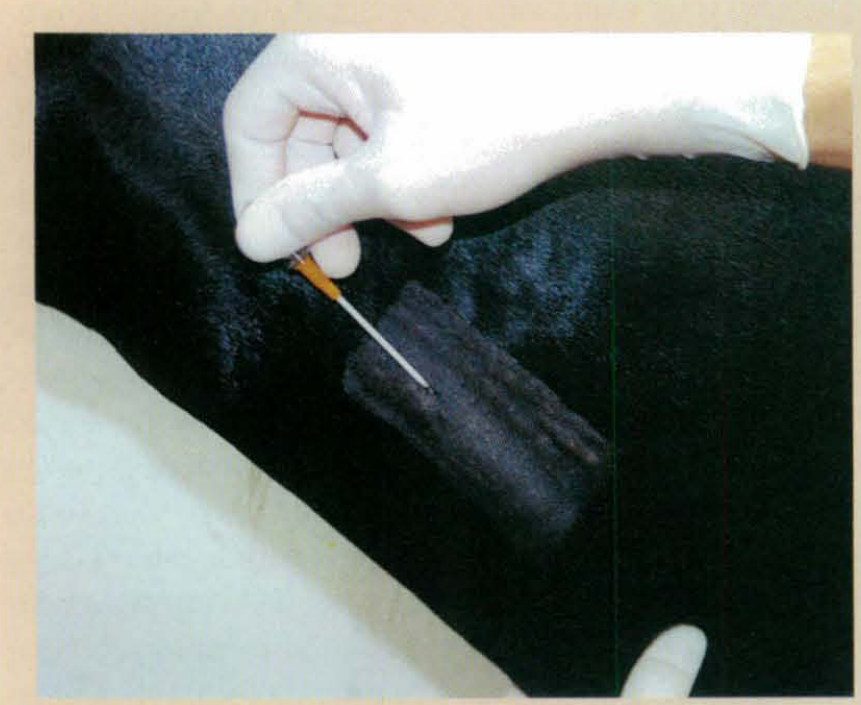

Figura 2. Posicionamento inicial do cateter sobre a veia jugular.

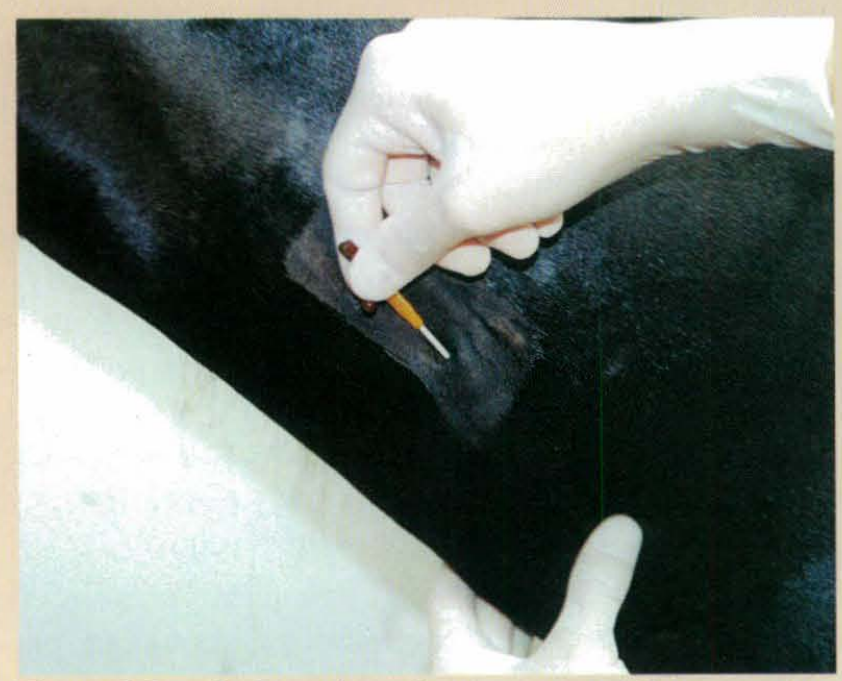

Figura 4. Introdução conjunta do cateter e mandril.

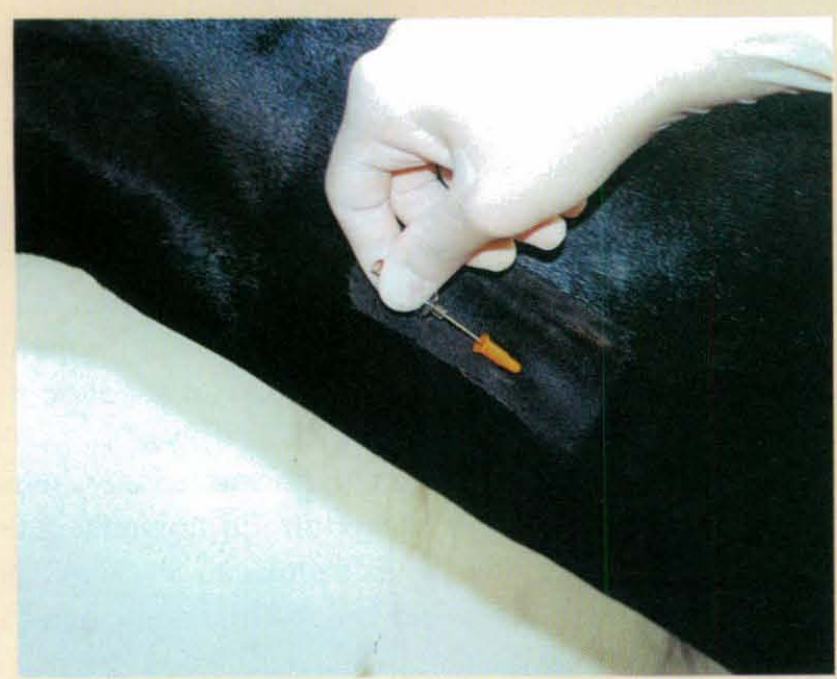

Figura 6. Remoção do mandril.

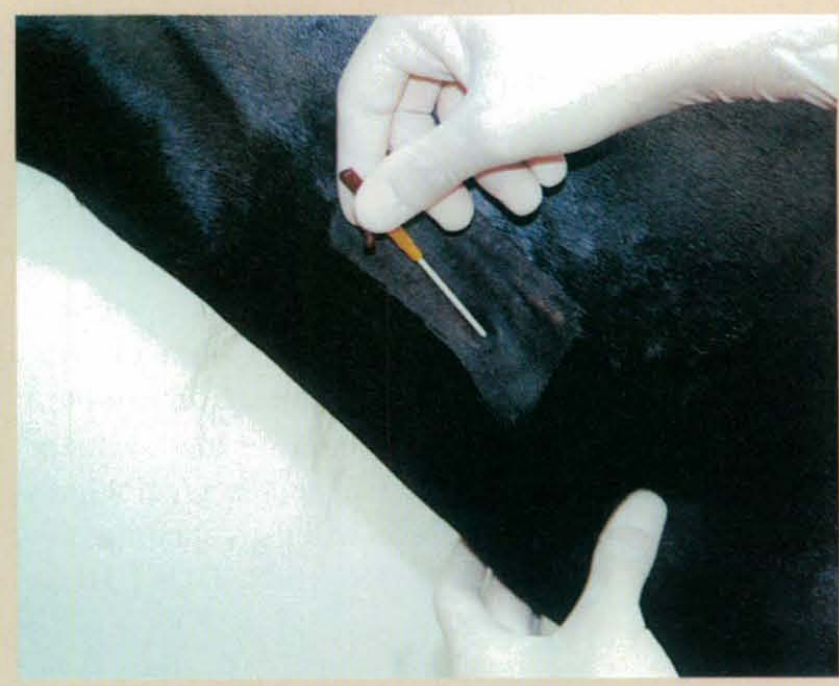

Figura 3. Venopunção inicial.

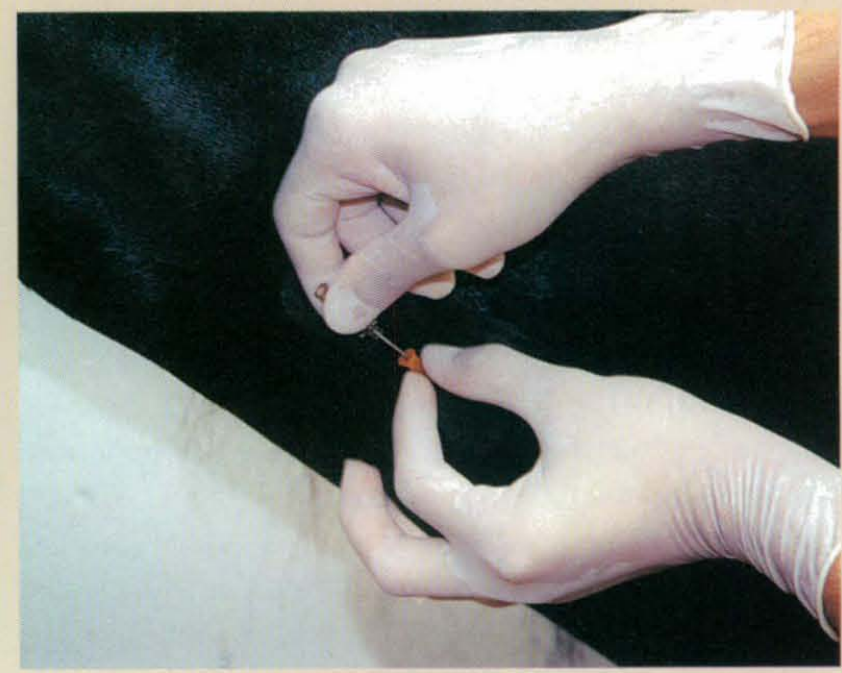

Figura 5. Deslizamento do cateter sobre o mandril imóvel.

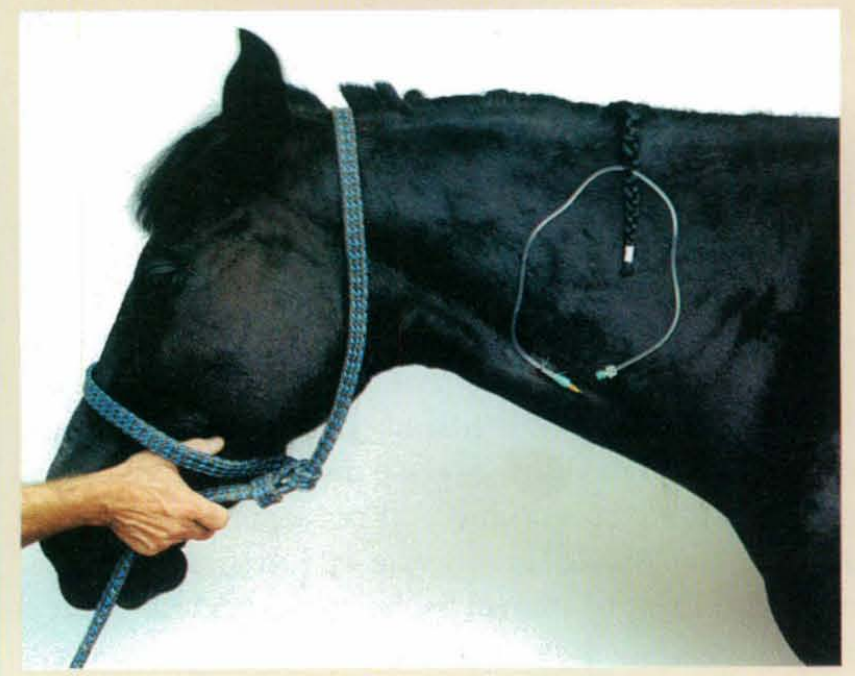

Figura 7. Acoplamento do tubo extensor ao cateter. 


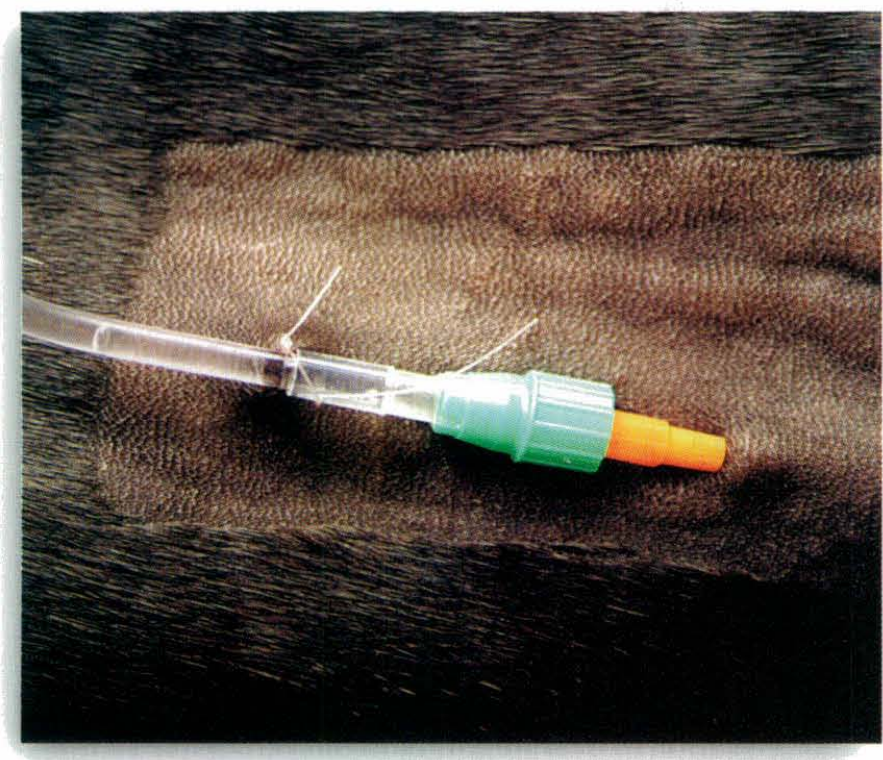

Figura 8. Fixação do cateter por meio de 2 pontos de sutura.

poder causar embolias, o coágulo formado no interior do cateter funciona como fonte potencial de infecção, uma vez que bactérias eventualmente introduzidas pelo lúmen permanecem aprisionadas na rede de fibrina do coágulo (BAYLY; VALE, 1982; WILLIAMSON, 1988).

O tempo de permanência do cateter é variável. De maneira geral, nenhum cateter deve permanecer posicionado na mesma veia por mais de 72 horas (BAYLY; VALE, 1982; WILLIAMSON, 1988), a não ser que sejam cateteres de uso prolongado (GEISER, 1993; TRAUB-DARGATZ, 1999). Caso haja necessidade de novo acesso intravenoso, cateterizar outra veia.

Todo o conjunto deve ser inspecionado periodicamente, no mínimo 1 vez ao dia, e na presença de sinais locais como hipertermia, aumento de volume e sensibilidade dolorosa, deve-se remover o cateter imediatamente (WILLIAMSON, 1988; SPIER et al., 1990; TRAUB-DARGATZ, 1999). A observação rigorosa às normas de higiene e anti-sepsia relativas não só à introdução, como também a todo o período de manutenção do cateter, é condição indispensável para se evitar a ocorrência de tromboflebites e septicemias (BAYLY; VALE, 1982).

Como já referido anteriormente, a velocidade de administração de fluidos depende da severidade das alterações clínicas num dado momento. Enquanto que situações emergenciais com grandes déficits de volume requerem a administração rápida de grandes quantidades de fluidos nas fases iniciais, situações menos críticas determinam uma abordagem menos agressiva. Iniciada a fluidoterapia, é imprescindível que o paciente seja monitorado clínica e laboratorialmente, se possível a intervalos regulares, para ajustar a velocidade de administração às novas condições de hidratação. Durante as fases iniciais da fluidoterapia pode ser preciso um monitoramento a cada 4 horas e à medida que ocorrem melhoras da condição orgânica, o monitoramento pode ser realizado a cada 12 a 24 horas (SEAHORN; CORNICK-SEAHORN, 1994). De modo geral, a velocidade máxima não deve ultrapassar 10 a $20 \mathrm{ml} / \mathrm{kg} / \mathrm{h}$, ou seja, 4 a 8 litros/ hora em um cavalo de $400 \mathrm{~kg}$ (SEAHORN; CORNICKSEAHORN, 1994); entretanto, em situações críticas de grandes déficits hidroeletrolíticos, velocidades superiores podem ser necessárias nas primeiras horas, mediante o uso de bombas de infusão e/ou acesso venoso auxiliar, quando são usadas, por exemplo, as duas veias jugulares. Deve-se estar atento ao fato de que velocidades de administração muito rápidas podem provocar diurese pela rápida expansão plasmática, sem, contudo, permitir a difusão do fluido administrado para outros compartimentos que, seguramente, apresentam déficits de volume (SPURLOCK; WARD, 1990; SEAHORN; CORNICK-SEAHORN, 1994; BARTON; MOORE, 1999).

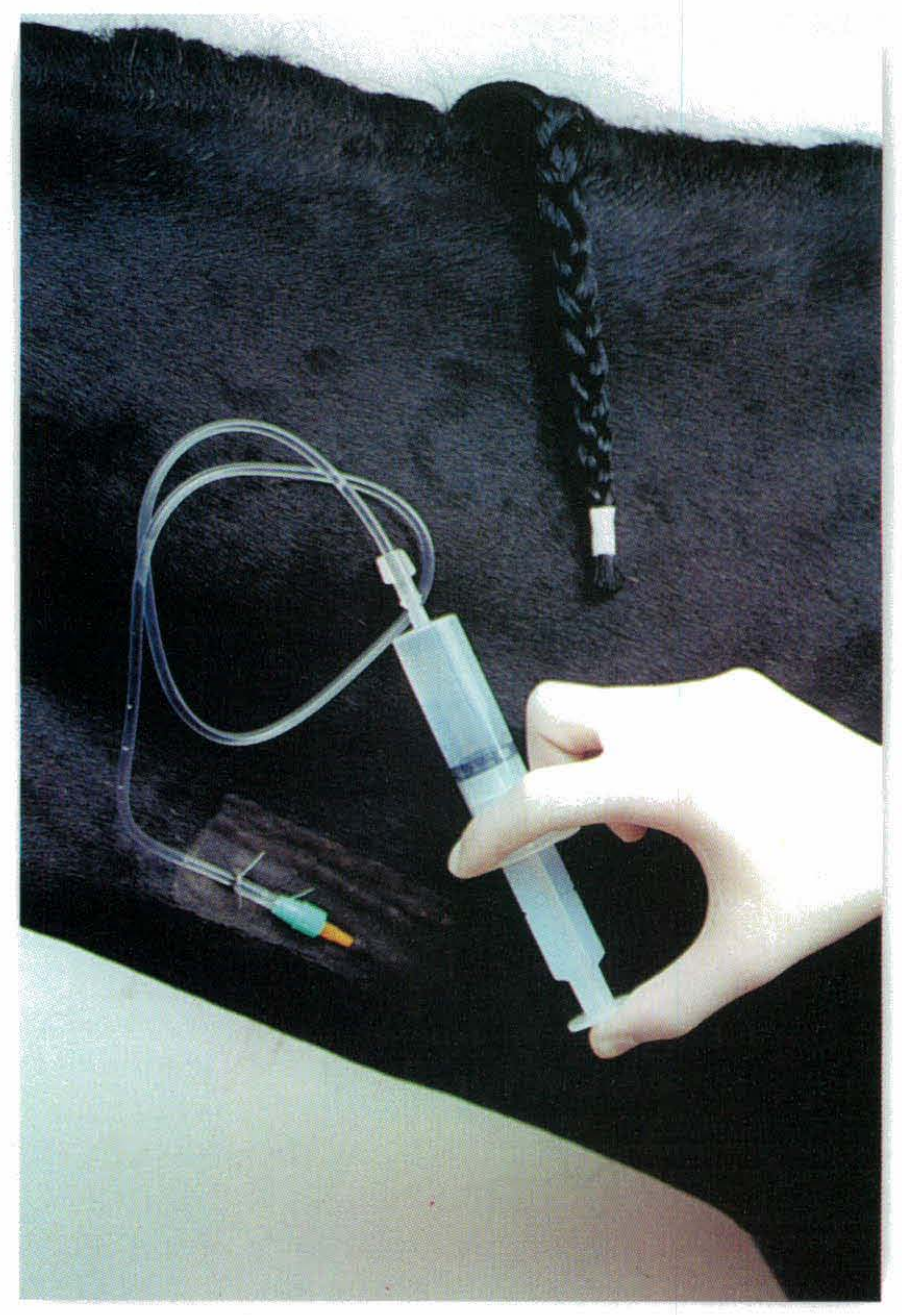

Figura 9. "Lavagem" do conjunto com solução heparinizada. 


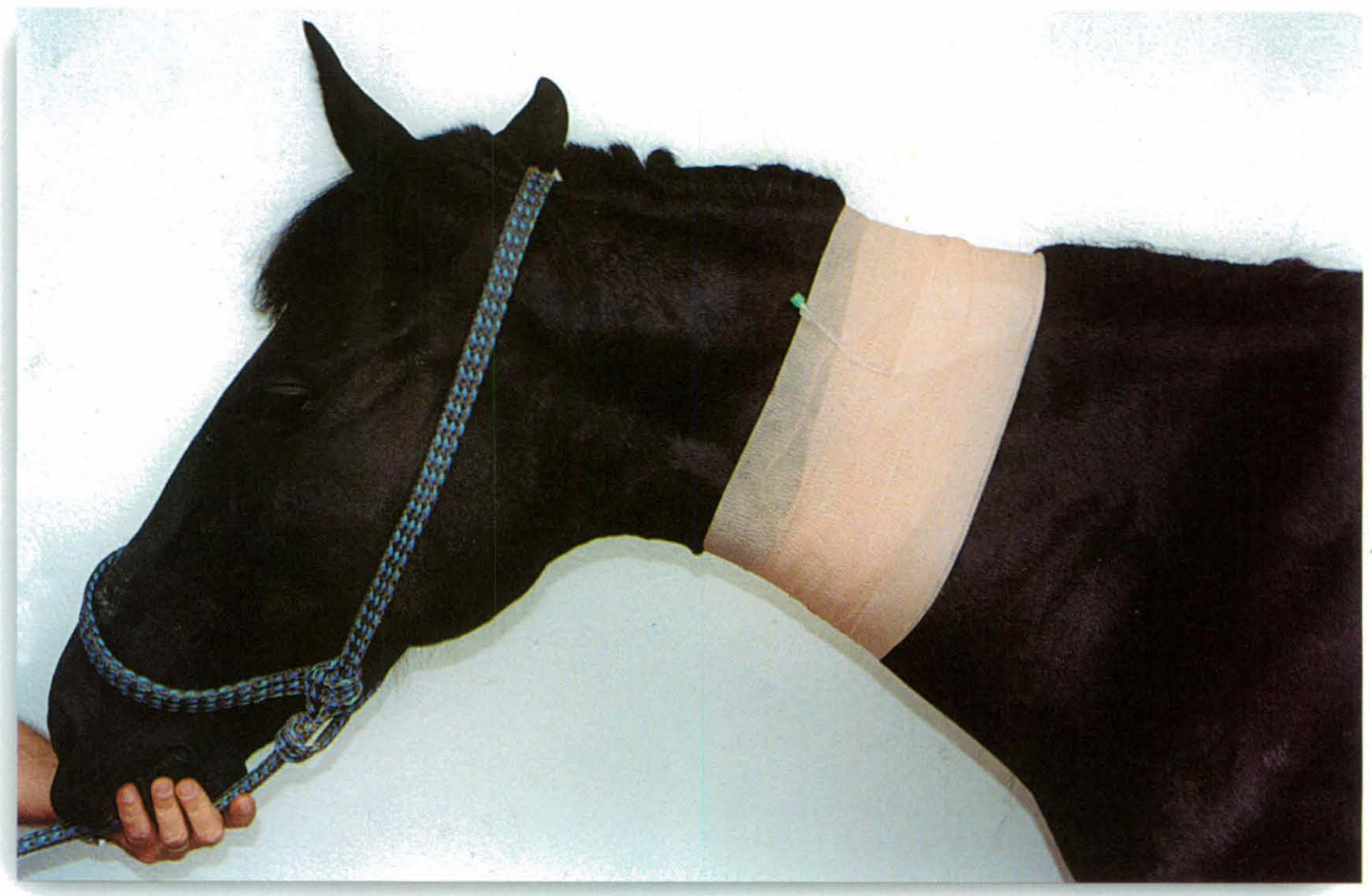

Figura 10. Recobrimento do local de venopunção com bandagem adesiva.

Inúmeras situações envolvendo desequilíbrios hidroeletrolíticos, tais como diarréias graves, seqüestro de fluidos no sistema digestório em cavalos portadores de cólicas obstrutivas e em animais que não estejam se alimentando, caracterizam-se por deficiências de potássio em diferentes graus. Considerando que o potássio é o principal íon responsável pelo equilíbrio osmótico no líqüido intracelular (LI) é que a hipocalemia severa pode resultar em distúrbios da condução neuromuscular esquelética e miocárdica, a suplementação de potássio deve ser instituída nessas situações (SCHMALL, 1992, 1997). Contudo, a administração deve ser cautelosa, uma vez que o excesso pode causar fibrilação ventricular seguida de parada cardíaca (GROSS, 1992).

Em virtude de a maior quantidade do potássio (98\%) concentrar-se no LI, a determinação sérica desse íon não representa com exatidão sua quantidade corpórea total (SPURLOCK; WARD, 1990; SCHMALL, 1992; SEAHORN; CORNICK-SEAHORN, 1994). Mesmo assim, esse valor pode servir como um guia para a reposição de potássio (SCHMALL, 1992).
Situações clínicas nas quais o valor se encontra abaixo de $3 \mathrm{mEq} / \mathrm{l}$ ou o cavalo esteja impossibilitado de ingerir alimentos podem ser suplementadas pela adição de potássio às soluções eletrolíticas na quantidade de 20 a $40 \mathrm{mEq} / \mathrm{l}$ por via intravenosa (SPIER et al., 1990; $\mathrm{SCH}$ MALL, 1992, 1997). A velocidade de administração não deve exceder a $0,5 \mathrm{mEq} / \mathrm{kg} / \mathrm{hora}$ (SPIER et al., 1990; SCHMALL, 1992, 1997; SEAHORN; CORNICK-SEAHORN, 1994). Caso se considere a via oral, a dose é de $40 \mathrm{~g} / 450 \mathrm{~kg}$ duas vezes ao dia. Tão logo o animal reassuma a ingestão de fibras, os níveis de potássio são prontamente restabelecidos (SPIER et al., 1990; SCHMALL, 1992, 1997).

A quantidade de bicarbonato a ser administrada pode ser calculada pela fórmula (HARTSFIELD, 1981; SPURLOCK; WARD, 1990; SEAHORN; CORNICK-SEAHORN, 1994; BARTON; MOORE, 1999):

$\mathrm{NaHCO}_{3}(\mathrm{mEq})=$ Déficit base $(\mathrm{mEq} / \mathrm{l}) \times 0,3 \times$ peso corpóreo $(\mathrm{kg})$.

O déficit de base é determinado pela gasometria. Considerando que $1 \mathrm{~g}$ de $\mathrm{NaHCO}_{3}$ possui $12 \mathrm{mEq}$ 
DEARO, A. C. O.; REICHMANN, P. Fluidoterapia em grandes animais - Parte II: quantidade e vias de administração / Fluid therapy in large animals - Part II: amount and administration routes / Rev. educ. contin. CRMV-SP / Continuous Education Journal CRMV-SP, São Paulo, volume 4, fascículo 3, p. 3 - 11, 2001.

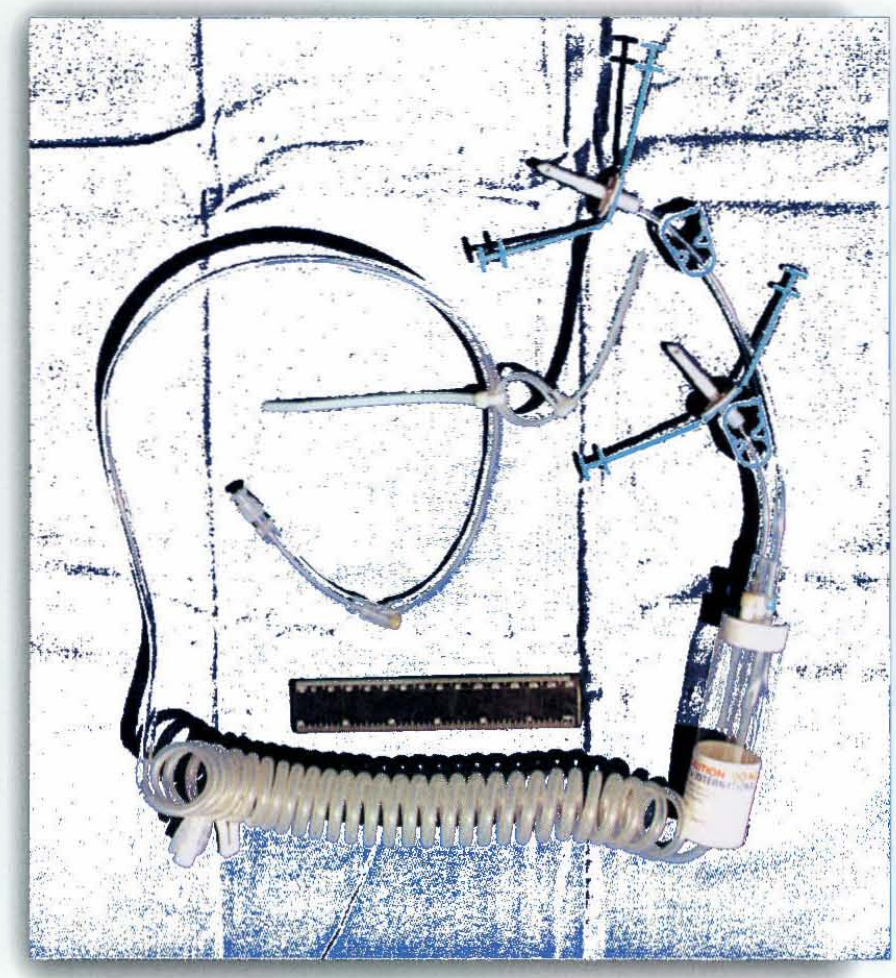

Figura 11. Equipo em espiral.

de $\mathrm{HCO}_{3}{ }^{-}$, a quantidade de bicarbonato de sódio total em gramas a ser administrada pode ser obtida dividindo-se a quantidade de bicarbonato de sódio calculada em $\mathrm{mEq}$ por 12. O volume final em litros de bicarbonato a ser administrado será calculado de acordo com a concentração da solução de bicarbonato de sódio disponível. Ao se tomar como exemplo um cavalo de 400 $\mathrm{kg}$ e que apresenta um déficit de base de $15 \mathrm{mEq} / \mathrm{l}$, tem-se:

$$
\text { NaHCO3 }(\mathrm{mEq})=15 \times 0,3 \times 400=1800
$$

$$
\begin{aligned}
& 1 \mathrm{~g} \mathrm{NaHCO}_{3} \\
& X-1800 \mathrm{mEq}
\end{aligned}
$$

Considerando-se que a concentração da solução de bicarbonato disponível é de 5\%, tem-se:

$$
\begin{aligned}
& 5 \mathrm{~g} \text { de } \mathrm{NaHCO}_{3} \text {-.-.- } 100 \mathrm{ml} \text { solução } \\
& 150 \mathrm{~g} \mathrm{-----} \mathrm{X} \quad X=3000 \mathrm{ml}=3 \text { litros }
\end{aligned}
$$

Portanto, são necessários 3 litros de uma solução de bicarbonato de sódio a $5 \%$ para a correção da acidose. Como precaução, deve-se administrar de $1 / 3$ a 1/2 do volume total de bicarbonato durante os primeiros $30 \mathrm{mi}$ nutos e reavaliar clínica e laboratorialmente o paciente. Caso não haja normalização do desequilíbrio, administrar o restante da solução nas próximas 12 a 24 horas de for-

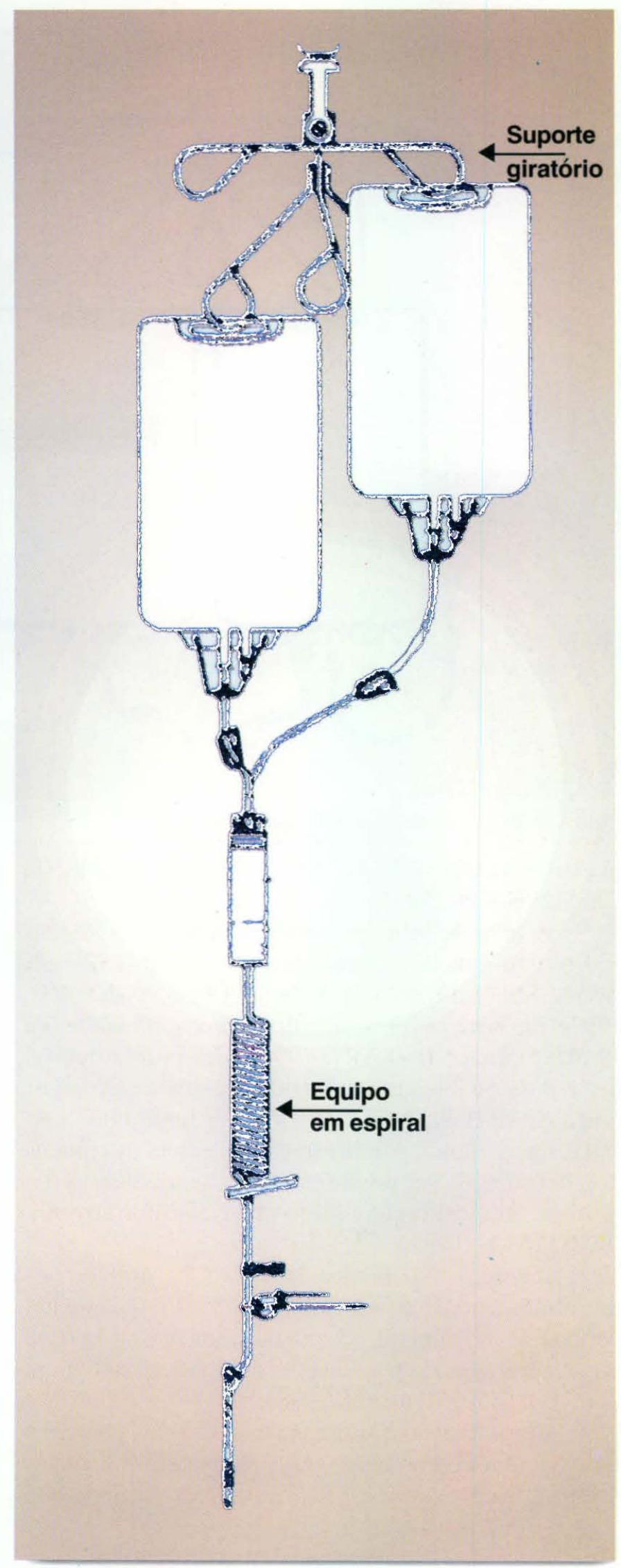

Figura 12. Suporte giratório e equipo em espiral. 


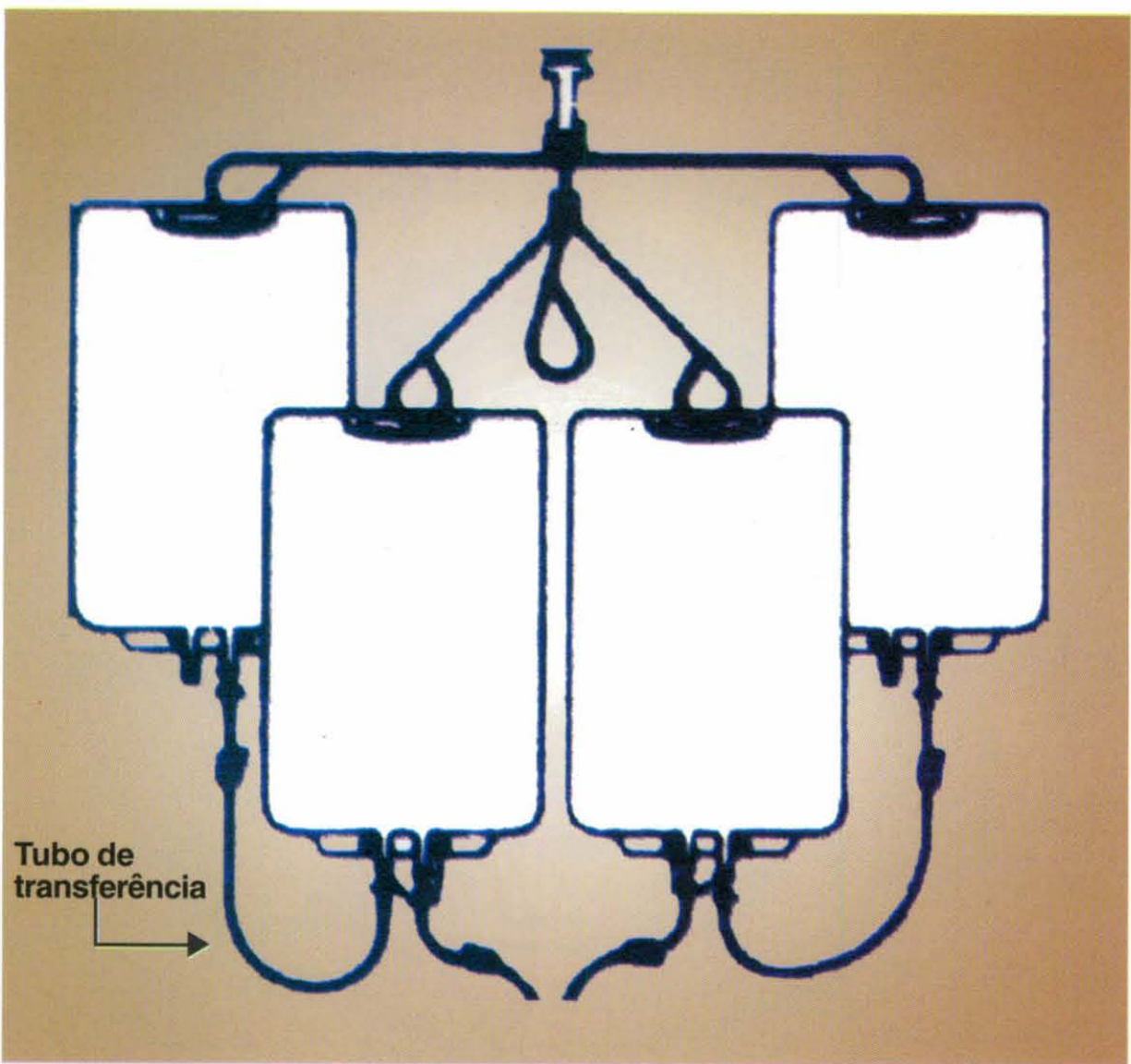

Figura 13. Bolsas plásticas e tubo de transferência.

ma lenta (HARTSFIELD, 1981; SEAHORN; CORNICK-SEAHORN, 1994).

A taxa de infusão venosa de glicose a $5 \%$ em eqüinos adultos deve permanecer entre 2 a $3 \mathrm{ml} / \mathrm{kg} / \mathrm{h}$ (ROUSSEL Jr.; KASARI, 1990; BARTON; MOORE, 1999). Em bovinos, deve-se utilizar uma taxa mais baixa (ROUSSEL Jr.; KASARI, 1990). Em potros neonatos, a dose de 3 a $5 \mathrm{ml} / \mathrm{kg} / \mathrm{h}$ pode ser usada com segurança (BARTON; MOORE, 1999). É importante ressaltar que a rápida administração de soluções contendo glicose pode ser prejudicial por causar diurese osmótica, desidratação, glicosúria e hiponatremia (SCHMALL, 1992).

A solução hipertônica de $\mathrm{NaCl} 7,5 \%$ deve ser administrada na dose de 4 a $6 \mathrm{ml} / \mathrm{kg}$ por via IV, durante um período de, no mínimo, 15 minutos. Sua aplicação deve ser sempre seguida por soluções eletrolíticas isotônicas (BERTONE, 1991; JEAN, 1998).

Considerando que em muitas situações clínicas a fluidoterapia deve estender-se por períodos prolongados, a utilização de material especial e volumes que permitem fluxo contínuo durante várias horas do dia reduz, em muito, a necessidade de acompanhamento ininterrupto. Em países como Estados Unidos e Canadá, a fluidoterapia prolongada é enormemente facilitada pelo uso de equipos especiais, em espiral, que garantem ampla movimentação do cavalo no interior da baia, e bolsas de 3 e 51 de soluções eletrolíticas balanceadas (Figuras 11 e 12). Esses produtos são fabricados especificamente para uso em animais de grande porte (INTERNATIONAL WIN, 2001). Os tubos de transferência, pelos quais o fluido pode ser transferido (até 20 litros, em uma única vez) de uma bolsa para outra sem sair do equipo que está acoplado ao tubo extensor (Figura 13), podem ser suspensos sobre a baia por meio de um suporte giratório e um sistema de rodízios e cordas, evitando, assim, a necessidade de repetidas intervenções para a reposição de bolsas adicionais

Outra grande vantagem decorrente do menor número de intervenções necessárias para a reposição de fluidos é a diminuição da ocorrência de contaminação da solução e de todo o sistema. Entretanto, um dos fatores limitantes do uso desse material é o custo elevado dos itens descartáveis como, por exemplo, o equipo em espiral que, para venda no mercado interno americano, custa US\$27.50 a unidade (INTERNATIONAL WIN, 2001).

No Brasil, até o presente momento, não é do conhecimento dos autores que esses materiais estejam disponíveis para comercialização no mercado interno, entretanto, formas alternativas de sistemas para fluidoterapia prolongada utilizando-se produtos nacionais são utilizados por alguns centros de forma artesanal ou improvisada. Embora não se disponha de soluções eletrolíticas para uso intravenoso em grandes volumes fabricadas em escala industrial, muitas soluções utilizadas rotineiramente na prática clínica podem ser formuladas em laboratórios apropriados, utilizando-se de recipientes com grande capacidade (KEMP, 1988a,b). 
DEARO, A. C. O.; REICHMANN, P. Fluidoterapia em grandes animais - Parte II: quantidade e vias de administração / Fluid therapy in large animals - Part II: amount and administration routes / Rev. educ. contin. CRMV-SP / Continuous Education Journal CRMV-SP, São Paulo, volume 4, fascículo 3, p. 3 - 11, 2001.

\section{SUMMARY}

The amount, administration routes and fluid flow rates are as important as the indications of fluid therapy and which fluid to use. Considering that fluid therapy is often maintained for long periods in many clinical situations, the use of intravenous catheters and large animal IV administration sets reduce the need for continuous supervision.

Key words: fluid therapy, dehydration, catheter, hydration, electrolytes

\section{REFERÊNCIAS}

1. BARTON, M. H.; MOORE, J. N. Fluid and electrolyte therapy. In: COLAHAN, P. T.; MAYHEW, I. G.; MERRITT, A. M. et al. Equine medicine and surgery. 5. ed. St. Louis: Mosby, 1999. cap. 4, p. 146-152.

2. BAYLY, W. M.; VALE, B. H. Intravenous catheterization and associated problems in the horse. Compendium on Continuing Education for the Practicing Veterinarian, v. 4, n. 5, p. 27-37, 1982.

3. BERTONE, J. J. Hypertonic saline in the management of shock in horses. Compendium on Continuing Education for the Practicing Veterinarian, v. 13, n. 4, p. 665-668, 1991.

4. DORNBUSCH, P. T.; HUSSNI, C. A.; THOMASSIAN, A. et al. Tromboflebite jugular nos eqüinos. Revista de Educação Continuada do CRMV-SP, v. 3, n. 2, p. 47-53, 2000.

5. GARDNER, S. Y.; DONAWICK, W. J. Jugular vein trombophlebitis. In: ROBINSON, N. E. Current therapy in equine medicine. 3. ed. Philadelphia: W.B. Saunders, 1992. cap. 9, p. 406-408.

6. GEISER, D. R. Wire-guided intravenous catheterization in the horse. Veterinary Medicine, v. 88, n. 6, p. 557-562, 1993.

7. GOLENZ, M. R.; CARLSON, G. P.; MADIGAN, J. E. et al. Preliminary report: The development of an intraosseous infusion technique for neonatal foals. Journal of Veterinary Internal Medicine, v. 7, n. 6, p. 377-382, 1993.

8. GROSS, D. R. Drogas que atuam no equilíbrio líquido e eletrolítico. In: BOOTH, N. H.; MCDONALD, L. E. Farmacologia e terapêutica em veterinária. 6 . ed. Rio de Janeiro: Guanabara Koogan, 1992. cap. 8, p. 427-438.

9. HARTSFIELD, S. M. Sodium bicarbonate and bicarbonate precursors for treatment of metabolic acidosis. Journal of the American Veterinary Medical Association, v. 179, n. 9, p. 914-916, 1981.

10. INTERNATIONAL WIN. Price schedules. Disponível em: <http:/ /www.InternationalWIN.com. Acesso em: 22 fev. 2001.

11. JEAN, K. Y. Clinical hypertonic saline solutions. In: WHITE, N. A.; MOORE, J. N. Current techniques in equine surgery and lameness. 2. ed. Philadelphia: W. B. Saunders, 1998. cap. 4, p. 15-19.

12. KEMP, D. T. A comprehensive guide to intravenous fluid therapy in horses. Veterinary Medicine, v. 83, n. 2, p. 193-212, 1988a.

13. KEMP, D. T. Preparing intravenous fluids for treatment of colic. In: GORDON, B. J.; ALLEN JR., D. Field guide to colic mana- gement in the horse. Lenexa: Veterinary Medicine, 1988b. cap. 13, p. 213-230.

14. LOPES, M. A. F.et al. Tratamento de compactação do cólon maior em eqüídeos com fluidoterapia enteral. Ciência Rural, v. 28, n. 3, p. $417-422,1998$.

15. McGINNESS, S. G.; MANSMANN, R. A.; BREUHAUS, B. A Nasogastric electrolyte replacement in horses. Compendium on Continuing Education for the Practicing Veterinarian, v. 18, n. 8, p. 942-949, 1996.

16. MORRIS, D. D. Medical therapy of colic. In: GORDON, B. J.; ALLEN JR., D. Field guide to colic management in the horse. Lenexa: Veterinary Medicine, 1988. cap. 12, p. 201-206.

17. ROSE, R. J. A physiological approach to fluid and electrolyte therapy in the horse. Equine Veterinary Journal, v. 13, n. 1, p. 7-14, 1981.

18. ROUSSEL JR.; A. J.; KASARI, T. R. Using fluid and electrolyte therapy to help diarrheic calves. Veterinary Medicine, v. 85, n. 3, p. 303-311, 1990.

19. SCHMALL, L. M. Fluid and electrolyte therapy. In: ROBINSON, N. E. Current therapy in equine medicine. 3. ed. Philadelphia: W.B. Saunders, 1992. cap. 3, p. 18-22.

20. SCHMALL, L. M. Fluid and electrolyte therapy. In: ROBINSON, N. E. Current therapy in equine medicine. 4. ed. Philadelphia: W.B. Saunders, 1997. cap. 16, p. 727-731.

21. SEAHORN, T. L.; CORNICK-SEAHORN, J. Fluid therapy. The Veterinary Clinics of North America. Equine Practice, v. 10, n. 3, p. 517-525, 1994.

22. SPIER, S. J.; SNYDER, J. R.; MURRAY, M. J. Fluid and electrolyte therapy for gastrointestinal disorders. In: SMITH, B. P. Large animal internal medicine. St. Louis: Mosby, 1990. cap. 30, p. 708-715.

23. SPURLOCK, S. L.; WARD, M. V. Fluid therapy for acute abdominal disease. In: WHITE, N. A The equine acute abdomen. Malvern: Lea \& Febiger, 1990. cap. 6, p. 160-172.

24. TRAUB-DARGATZ, J. L. Intravenous catheterization techniques. In: COLAHAN, P. T.; MAYHEW, I. G.; MERRITT, A. M. et al. Equine medicine and surgery. 5. ed. St. Louis: Mosby, 1999. cap. 4, p. 136-146.

25. WILLIAMSON, L. Diagnostic procedures for evaluating equine colic. In: GORDON, B. J.; ALLEN JR., D. Field guide to colic management in the horse. Lenexa: Veterinary Medicine, 1988. cap. 7, p. 141-156. 